\title{
Acute Kidney Injury: Resurgence of Its Importance as a Cause of Chronic Kidney Disease
}

Acute kidney injury (AKI) has always been one of the most intriguing topics in nephrology with its ever-increasing incidence worldwide despite best efforts to curtail it by the nephrology community. AKI has an incidence of approximately 13.3 million persons per year and $85 \%$ of whom reside in the low or medium resource countries.

AKI carries a high mortality rate ( $>2.3$ million deaths worldwide every year), 3-fold increased cost of resources in AKI patients and the development of chronic kidney disease in $>30 \%$. $^{2}$ Therefore, it remains an area of ongoing high quality research to date but yet lack standardization possibly due to population differences and healthcare workers' lack of proper education and awareness of AKI. This is quite aptly highlighted throughout the first to $20^{\text {th }}$ Acute Dialysis Quality Initiative (ADQI) consensus conferences, Acute Kidney Injury Network (AKIN) initiative as well as Kidney Disease Improving Global Outcomes (KDIGO) consensus statements. ${ }^{2,3}$ Apart from the disparity in standardization of criteria of AKI, there has been considerable debate regarding lack of inclusion of aetiology and need for renal replacement therapy (RRT) within different classifications devised to date. ${ }^{4}$

The difference in etiology of AKI amongst the developed and developing countries add further disparity to reaching a consensus in prevention and management as highlighted by International Society of Nephrology AKIOby25 initiative. ${ }^{1}$ The marked difference in morbidity and mortality between community acquired AKI (CA-AKI) predominantly occurring in developing countries and hospital acquired $\mathrm{AKI}(\mathrm{HA}-\mathrm{AKI})^{7}$ and $\mathrm{AKI}$ in intensive care unit predominantly occurring in developed countries further complicates the issue.

7 CA-AKI is a result of infections, diarrhea, obstetric causes and snake bite whereas HA-AKI aetiology mainly includes hospital acquired infections, drug toxicity, systemic disease complications and multiorgan failure secondary to myocardial infarction or sepsis. ${ }^{8-11}$

The current issue of PJKD publishes a retrospective study by Habib et al determining the causes and outcomes of AKI in patients of different age groups, requiring hospitalization at a tertiary care hospital in a developing country. ${ }^{12}$ This study extrapolates AKI classification based on the causes, age and outcome. The study conforms to previous studies data highlighting Pregnancy related AKI (P-AKI) to be on the top. However, despite high dialysis institution, majority of patients recovered completely with low mortality. Furthermore, an unusually high number of obstructive uropathy was reported in this study. Quite remarkably CKD conversion was only $26 \%$ in this study. This data where highlights the acuteness of the disease state at the same time is encouraging in terms of the outcomes.

Recently the American Society of Nephrology has proposed, AKI!Now, an initiative to promote excellence in preventing and managing AKI with goals to transform education and delivery of AKI care, to decrease morbidity and mortality, and to improve long-term outcomes. ${ }^{13}$ It further proposes to include 'prerenal AKI' and 'subclinical AKI' in the already existing classifications of AKI in order to underscore causality and pathophysiology in the understanding and management of AKI under different settings and different populations. This quality initiative takes into account lack of awareness of AKI amongst healthcare teams especially in the developing world.

There has been considerable research in the field of novel biomarkers for early diagnosis of AKI but due to higher cost they have limited applicability to only research centres. ${ }^{14}$ In our part of the world limited number of studies have been published but have proven to be more of an audit rather than adding any significant knowledge to the AKI research pool in terms of management and largely highlights lack of awareness in the public as well as healthcare teams. ${ }^{15}$ Recent COVID 19 has also increased interest in AKI pathophysiology due to novel viral diseases and results of ongoing research worldwide is eagerly awaited as it poses significant mortality risk to COVID 19 patients.

The way forward remains open to exploration and resolution of existing controversies in AKI with regards to risk assessment, diagnosis, fluid management, hemodynamic management, drug stewardship and RRT.

Wide variation exists within local as well as international centres in adaptation of new technologies as many still follow a conservative approach to AKI management and is a sufficient justification for Revisiting the 2012 KDIGO AKI guidelines. ${ }^{17}$ In Pakistan, we need to develop a digital database for reporting AKI as well as to envision a government initiative for provision of education/awareness to the public, healthcare workers and policy makers in order to reduce morbidity and mortality resulting from AKI. In this respect the current study by Habib et.al. is a sign of renewed interest in AKI and we look forward for further publications.

\section{References}

1. Mehta R L et al. International Society of Nephrologys Oby25 initiative for acute kidney injury (zero preventable deaths by 2025): A human rights case for nephrology. Lancet. 2015;385(9987):2616-43. 
2. Mehta R, Bagga A, Patibandla R, Chakravarthi R. Detection and Management of AKI in the Developing World: The 18th Acute Disease Quality Initiative (ADQI) International Consensus Conference. Kidney Int Rep. 2017;2(4):515 - 8.

3. Lopes J A, Jorge S. The RIFLE and AKIN classifications for acute kidney injury: a critical and comprehensive review. Clin Kidney J. 2013;6(1):8-14.

4. Kher V, Srisawat N, Noiri E, Benghanem Gharbi M, Shetty MS, Yang L, Bagga A, Chakravarthi R, Mehta R; Acute Disease Quality Initiative (ADQI) Consensus Group. Prevention and Therapy of Acute Kidney Injury in the Developing World. Kidney Int Rep. 2017;2(4):544 - 58.

5. Kaaviya R et al. Community Acquired AKI: A Prospective Observational Study from a Tertiary Level Hospital in Southern India. Indian J Nephrol.2019; 29(4): 254-60

6. Jha V, Chugh K S. Community-Acquired Acute Kidney Injury in Asia. Semin Nephrol. 2008; 28(4):330-347.

7. Mahbub T et al. Etiology and Short term Outcome of Acute Kidney Injury (AKI) in Hospitalized Patients: A Single Center Study. Bangladesh Crit Care J. 2019; 7(2): 77-80.

8. Javaid B, Adil N, Munawar W. Factors Leading to Acute Kidney Injury in Third Trimester of Pregnant Females Visiting Allied Hospital Faisalabad. Ann Punjab Med Coll. 2018;12(4):288-90.

9. Khattak M I, Sabir S, Khattak S A, Tahir N. Spectrum of Acute Kidney Injury (AKI) in Pregnancy. J Rawal Med Coll. 2014;18(2):267-9.

10. Khan I et al. Frequency of acute kidney injury and its short-term effects after acute myocardial infarction. J Pak Med Assoc. 2017; 67(11): 1693-97

11. Khan SA, Mirbahar A, Qadir M. Acute kidney injury in hospitalized patients frequency of various etiologies. Prof Med J 2019;26(11):1952-57

12. Liu KD, Goldstein SL, Vijayan A, Parikh CR, Kashani K, Okusa MD et.al. AKI!Now Initiative: Recommendations for awareness, recognition, and management of AKI. Clin J Am Soc Nephrol 2020;15(12):1838-1847

13. Basu RK, Gist K, Wheeler DS: Improving acute kidney injury diagnostics using predictive analytics. Curr Opin Crit Care 2015;21:473-78.

14. Ponce D, Balbi A. Acute kidney injury: risk factors and management challenges in developing countries. Intl J Nephrol Renovasc Dis 2016:9: 193-200.

15. Batlle D et al. Acute Kidney Injury in COVID-19: Emerging Evidence of a Distinct Pathophysiology. J Am Soc Nephrol 2020;31(7):1380-83

16. Ostermann M, Bellomo R, Burdmann EA, Doi K, Endre ZH, Goldstein SH et al. KDIGO Executive Conclusions: Controversies in acute kidney injury: conclusions from a Kidney Disease: Improving Global Outcomes (KDIGO) Conference report. Kidney Internation 2020;98: 294 - 309

\author{
Brig Sohail Sabir \\ Professor of Medicine, \\ Army Medical College Rawalpindi \\ Head of Transplant Nephrology, \\ Armed Forces Institute of Urology Rawalpindi, Pakistan \\ Personal Physician to the President of Islamic Republic of Pakistan \\ Email: drsohailsa@hotmail.com
}

